\title{
Alcohol and cardiovascular mortality crisis in Russia
}

\author{
Razvodovsky YE* \\ Grodno State Medical University, 80 Gorky Street, Grodno 230009, Republic of Belarus
}

Cardiovascular disease (CVD) remains the leading cause of death in Europe and despite recent decreases in mortality rates in many countries, it is still accounting for over 4.35 million deaths each year [1]. The burden of CVD in Europe varies dramatically between countries. Russia has one of the highest CVD morbidity and mortality rates, accounting for $57 \%$ of all deaths in the country [2]. In Russia, premature CVD mortality was estimated to cost 24,400.4 million Euros in 2009 [3]. The main diseases that define a high level of mortality from CVD are coronary heart disease (CHD) and cerebrovascular diseases, which are responsible for 49.3 percent and 35.3 percent of deaths, respectively [4]. A distinctive feature of CVD mortality in Russia is that the high rates occur in working age population, especially in males [5].

High mortality from CVD in Russia and its profound fluctuations over the past decades have attracted considerable interest. It was repeatedly emphasized that phenomenon of high CVD mortality in Russia is only partially associated with the traditional CVD risk factors identified in epidemiologic studies [2,6]. It was suggested that alcohol provides the most plausible explanation for both the high rate and the dramatic fluctuation in Russian CHD mortality rate during the last decades. Some experts have underlined the importance of binge drinking as the main reason for the CVD mortality crisis in Russia [79]. There is direct individual-level evidence linking excessive drinking and CVD in Russia. A case-control study of men aged 20-55 years in the major cities of the Udmurd Republic in 1998-1999 found that history of heavy drinking in the past few years is strongly associated with risk of death from CVD (OR=2.42;CI:2.35-7.55) [10]. These findings provided first individual-level evidence in support of the hypothesis that binge drinking is key to explaining the heavy burden of CVD mortality in Russia.

Another prospective cohort study of 6502 men aged 25-64 years in Novosibirsk showed that frequent heavy drinking, defined as drinking at least three times a week in a dose greater than $120 \mathrm{~g}$ of ethanol per occasion, is significantly associated with incidence of cardiovascular mortality compared with moderate drinking $(\mathrm{RR}=2,05: 1,69-3,86)[11]$. A more recent large retrospective study in three typical Russian cities (Barnaul, Byisk and Tomsk) based on proxy information on alcohol consumption from families of 48557 adults who had died in 19902001 had found strong dose-response association between alcohol consumption and CVD mortality [12]. Several CVD codes were strongly associated with heavy drinking (3 or more bottles of vodka per week) in men: acute ischemic heart disease other than myocardial infarction $(\mathrm{RR}=3.04 ; \mathrm{CI}: 2.73-3.39)$ and chronic ischemic heart disease $(\mathrm{RR}=1.49 ; \mathrm{CI}: 1.33-1.68)$. Cardiovascular diagnoses were even more strongly associated with alcohol in women: acute myocardial infarction $(\mathrm{RR}=2.04 ; \mathrm{CI}: 1.59-2.61)$, acute ischemic heart disease other than myocardial infarction ( $\mathrm{RR}=9.29 ; \mathrm{CI}: 7.97-10.74)$, chronic ischemic heart disease $(\mathrm{RR}=2.58$; $\mathrm{CI}: 2.09-3.18)$ and stroke $(\mathrm{RR}=1.62 ; \mathrm{CI}: 1.37-1.93)$. It was also reported that acute ischemic heart disease other than myocardial infarction includes same misclassified deaths that were actually from alcohol poisoning and was the largest single contributor to the alcohol-associated excess mortality [12].

A prospective cohort study of 1966 men and 1738 women performed in Arkhangelsk in 1999-2000 reported a significant dose-response association between hazardous alcohol consumption and the risk of cardiovascular death in women [13]. Women reporting a consumption of $80 \mathrm{~g}$ alcohol or more at least monthly and a consumption of 5 alcohol units or more on one drinking episode had higher risk of cardiovascular death than abstainers (RR was 5.06;CI:1.54-16.7 and 3.21:CI:1.07-9.58, respectively). An average increase in one score on the scale of AUDIT increased risk of cardiovascular death by $26 \%$; the corresponding risk estimate for CAGE was higher (RR 2.46 per increase in one score). A positive BAC was found in $36 \%$ of male and $30 \%$ of female deaths. This was the first cohort study from Russia where strong association between binge drinking and the risk of cardiovascular death was found in women.

Several Russian studies used autopsy data to examine the association between alcohol consumption and CVD mortality. Two of them were conducted in Izhevsk and were focused on deaths among men of working age. The first study, in which postmortem blood alcohol concentration were measured in men aged 20-55 showed that $38.2 \%$ of those recorded as dying from CVD were BAC-positive at time of deaths [14]. Medium level of alcohol intoxication occurred in $24.7 \%$ of those recorded as dying from CVD disease. BAC levels consistent with strong intoxication were seen in $13.5 \%$ of deaths from CVD. The authors concluded that the high deaths rates from CVD in Russia cannot be explained by misclassification of deaths attributable to acute alcohol poisoning as no men dying from CVD had blood alcohol concentration in excess of $400 \mathrm{mg} / \mathrm{dl}$ [14].

Another case-control study of mortality among working-age (2554 years) men occurring in the city of Izhevsk reported that hazardous drinking was associated with an increased risk of death from CVD (OR=4.14;CI:3.23-5.31) adjusted for age, smoking and education [15]. Although there was a strong association with $\mathrm{CHD}(\mathrm{OR}=4.04$; $\mathrm{CI}$ : 2.795.84), the association with alcoholic cardiomyopathy was particularly strong ( $\mathrm{OR}=15.7$; CI: 9.5-25.9). Furthermore, the mortality from CVD was strongly associated with markers of alcohol-related problems such as registration with the alcohol treatment service (OR=3.71; CI: 2.52-5.47), proxy report of hazardous drinking over the preceding year $(\mathrm{OR}=4.14$; $\mathrm{CI}: 3.23-5.31)$ and proxy reports of whether or not an

Correspondence to: Razvodovsky YE, Grodno State Medical University, 80 Gorky Street, Grodno 230009, Republic of Belarus, Tel: + 37501527018 84, Fax: +3750152 4353 41, E-mail: razvodovsky@tut.by

Received: April 14, 2016; Accepted: May 04, 2016; Published: May 09, 2016 
extended binge drinking episode (zapoi) occurred in the previous week $(\mathrm{OR}=9.62$; CI: 6.2-14.91). In this study alcoholic cardiomyopaty deaths comprised $20 \%$ of all CVD deaths. Based on this evidence the authors have concluded that the positive association of chronic harmful drinking resulting in alcoholic cardiomyopathy and acute effects of binge drinking [15].

The most recent study on the association between alcohol use before deaths and CVD mortality in the city of Arkhangelsk, Northwest Russia, identified alcohol consumption before death as an important correlate of premature CVD mortality [16]. About one third of men and women who died from CVD under 60 had consumed alcohol shortly before deaths. The highest proportion of BAC-positive deaths was seen among those who died from cardiomyophaties and the two CHD subgroups "other acute or sub acute cardiac ischemia" and atherosclerotic heart disease". Alcoholic cardiomiopathy is an important component of premature CVD mortality in Arkhangelsk. It was responsible for the one third and one fifth of all female and male CVD deaths under age of 50 years. The authors found little evidence of misclassification of deaths from acute alcohol poisoning as cardiovascular deaths: only $5 \%$ of male and $1 \%$ of female deaths classified as being due to CVD has high BACs $(2.5$ to $3.99 \mathrm{~g} / \mathrm{l})[16]$.

The role of excessive drinking pattern as a powerful risk factor for CVD mortality was emphasized in a study based on data from Moscow City that reported about increased in deaths from alcohol poisoning and cardiovascular disease on weekends reflecting weekend binge drinking pattern [17]. Similar findings were reported in a more recent population-based study which assessed daily variations in ambulance calls for CVD in Arkhangelsk in 2000-2008 [18]. There was a significant daily variation in calls for CVD in men and women aged 18-59, with increased numbers of calls on weekends and Mondays. The authors hypothesize that these variations might be associated with excessive drinking on the weekends. These findings support the argument that binge drinking pattern may potentiate negative role of alcohol as major cause of high cardiovascular death rate in Russia.

It was hypothesized that in Russia, unspecified CVD codes mask large number of deaths from acute alcohol poisoning [10]. This hypothesis is supported by the sharp fluctuations in mortality attributed to other acute IHD aged 35-69 years during 1990-2006, while there was little variation in the mortality attributable to acute myocardial infarction [14]. Furthermore, in a survey of forensic autopsies in Barnaul during 1990-2004 at which post-mortem blood alcohol was recorded, $15 \%$ of those who died aged 15-69 years with non-myocardial infarction acute IHD had BAC over $4 \mathrm{~g} / \mathrm{L}$, which is potentially lethal [12]. However, this hypothesis was not supported by more recent studies [16].

In a number of studies using various designs alcohol consumption has been found to be important risk factor of CVD mortality in Russia. The high level of alcohol consumption in combination with detrimental drinking pattern in Russia results in high level of CVD mortality and strong individual-level association between alcohol consumption and CVD mortality. Accumulated research and empirical evidence suggest that any attempts to reduce CVD mortality burden in Russia should be linked with efforts through restriction of availability of alcohol.

\section{References}

1. Rayner M, Allender S, Scarborough P (2009) British Heart Foundation Health Promotion Research Group Cardiovascular disease in Europe. Eur J Cardiovasc Prev Rehabil 16 Suppl 2: S43-47.[Crossref]

2. Kim AS, Johnston SC (2011) Global variation in the relative burden of stroke and ischemic heart disease. Circulation 124: 314-323.[Crossref]

3. Kontsevaya A, Kalinina A, Oganov R (2013). Economic Burden of Cardiovascular Diseases in the Russian Federation. Health Regional Issues 2:199 - 204.

4. Pajak A, Kozela M (2012). Cardiovascular disease in Central and Eastern Europe. Public Health Review 33: 416-435.

5. Petruchin IS, Lunina EY(2012). Cardiovascular disease risk factors and mortality in Russia: challenges and barriers. Public Health Review 2: 436-449

6. Averina M, Nilssen O, Brenn T, Brox J, Kalinin AG, et al. (2003) High cardiovascular mortality in Russia cannot be explained by the classical risk factors. The Arkhangelsk Study 2000. Eur J Epidemiol 18: 871-878.[Crossref]

7. Britton A, McKee M (2000) The relation between alcohol and cardiovascular disease in Eastern Europe: explaining the paradox. J Epidemiol Community Health 54: 328332.[Crossref]

8. McKee M, Britton A(1998). The positive relationship between alcohol and hear disease in Eastern Europe: potential physiological mechanisms. J R Soc Med 91:402-7.

9. Razvodovsky YE (2009)Alcohol poisoning and cardiovascular mortality in Russia 1956-2005. Alcoholism 1: 27-42.

10. Shkolnikov V, Chervyakov VV, McKee M, Leon DA (2002) Russian mortality beyond vital statistics. Effects of social status and behaviors on deaths from circulatory disease and external causes - a case-control study of men aged 20-55 years in Urdmurtia, 1998-99 Paper presented at the IUSSP Seminar "Determinants of Diverging Trends in Mortality", Rostock, June 19-2.

11. Malyutina S, Bobak M, Kurilovitch S, Gafarov V, Simonova G, et al. (2002) Relation between heavy and binge drinking and all-cause and cardiovascular mortality in Novosibirsk, Russia: a prospective cohort study. Lancet 360: 1448-1454.[Crossref]

12. Zaridze D, Maximovitch D, Lazarev A, Igitov V, Boroda A, et al. (2009) Alcohol poisoning is a main determinant of recent mortality trends in Russia: evidence from a detailed analysis of mortality statistics and autopsies. Int J Epidemiol 38: 143-153. [Crossref]

13. Sidorenkov O, Nilssen O, Grjibovski AM (2012) Determinants of cardiovascular and all-cause mortality in northwest Russia: a 10-year follow-up study. Ann Epidemiol 22: 57-65.[Crossref]

14. Shkolnikov VM, McKee M, Chervyakov VV, Kyrianov NA(2002). Is the link between alcohol and cardiovascular death among young Russian men attributable to misclassification of acute alcohol intoxication? Evidence from the city of Izhevsk. $J$ Epidemiol Community Health. 56:171-175

15. Leon DA, Saburova L, Tomkins S, Andreev E, Kiryanov N, et al. (2007) Hazardous alcohol drinking and premature mortality in Russia: a population based case-control study. Lancet 369: 2001-2009.[Crossref]

16. Sidorenkov O, Nilssen O, Nieboer E, Kleshchinov N, Grjibovski AM(2011). Premature cardiovascular mortality and alcohol consumption before death in Arkhangelsk, Russia: an analysis of a consecutive series of forensic autopsies. Int $J$ Epidemiol 40:1519-29.

17. Chenet L, McKee M, Leon D, Shkolnikov V, Vassin S (1998) Alcohol and cardiovascular mortality in Moscow; new evidence of a causal association. J Epidemiol Community Health 52: 772-774.[Crossref]

18. Zaridze D, Maximovitch D, Lazarev A, Igitov V, Boroda A, et al. (2009) Alcoho poisoning is a main determinant of recent mortality trends in Russia: evidence from a detailed analysis of mortality statistics and autopsies. Int J Epidemiol 38: 143-153. [Crossref]

Copyright: (C2016 Razvodovsky YE. This is an open-access article distributed under the terms of the Creative Commons Attribution License, which permits unrestricted use, distribution, and reproduction in any medium, provided the original author and source are credited. 